\title{
Value of Hysteroscopy and Transvaginal Sonography in Endometrial Pathology in Bleeding and Nonbleeding Postmenopausal Women
}

\author{
Poonam Mani ${ }^{1}$, Lalita Yadav ${ }^{2}$, Arushi Singh ${ }^{3}$, Tuheena Gupta ${ }^{4}$
}

\begin{abstract}
The evaluation of the endometrium has evolved with varying amounts of evidence and often little validation of what has dominated the clinical practice.

Aims and objectives: To study the value of hysteroscopy and transvaginal sonography (TVS) in endometrial pathology in bleeding and nonbleeding postmenopausal women.

Materials and methods: A prospective study was conducted in the Department of Obstetrics and Gynecology, Subharti Medical College, Meerut, Uttar Pradesh, India. A total of 280 menopausal patients with or without postmenopausal bleeding (PMB) were included in our study. Out of 280 women, 204 women who had PMB were put in group I, and 76 women with no PMB were put in group II after exclusion criteria were met. Results: In our study, majority (46.6\%) had polyp, 9.47\% had atrophic endometrium, $29.29 \%$ had normal endometrium, $2.4 \%$ women had endometrial hyperplasia, and $2.1 \%$ had endometrial carcinoma on histopathology, which positively correlated with hysteroscopy. For diagnosing any pathology, hysteroscopy had better specificity $(p<0.001)$, although the two methods did not differ with regard to sensitivity $(p=0.188)$. For the diagnosis of polyps of any size, hysteroscopy had better sensitivity $(p<0.001)$; however, specificities did not differ $(p=1.0)$.

Conclusion: There seems to be great confusion about appropriate evaluation in symptomatic menopausal women using TVS and/or hysteroscopy as well as how to proceed with incidental findings in asymptomatic postmenopausal women. A significant number of patients without PMB had abnormal findings on hysteroscopy like polyps. However, none had malignancy in this group.

Keywords: Endometrium, Hysteroscopy, Postmenopausal bleeding, Transvaginal sonography.

Journal of South Asian Federation of Obstetrics and Gynaecology (2020): 10.5005/jp-journals-10006-1769
\end{abstract}

\section{INTRODUCTION}

Menopause is the permanent cessation of menstruation resulting from the loss of ovarian follicular activity. ${ }^{1}$

Bleeding that occurs 12 months after the menopause is called postmenopausal bleeding (PMB) and needs evaluation. Even nonbleeding symptomatic uterine conditions, other risk factors, and incidental findings on clinical or transvaginal sonography (TVS) screening studies require an evaluation of the uterine cavity. Hysteroscopy permits the direct visualization and assessment of endocervical and uterine cavities. ${ }^{2}$

However, hysteroscopy is not as cost-effective as TVS, which is associated with relatively less patient discomfort and does not require anesthesia. However, these currently available modalities are far from being perfect.

Decisions about further investigations should be made on a case-by-case basis in asymptomatic women with increased endometrial thickening and risk factors for endometrial cancer such as obesity, hypertension, diabetes, and late menopause.

In asymptomatic women on tamoxifen, a routine ultrasound for endometrial thickening should not be performed.

Not all postmenopausal women who have asymptomatic endometrial polyps require surgery. Women found to have asymptomatic polyps on ultrasound should be triaged for intervention according to size of the polyp, age, and other risk factors. ${ }^{3}$

\footnotetext{
${ }^{1-4}$ Department of Obstetrics and Gynecology, Subharti Medical College, Meerut, Uttar Pradesh, India

Corresponding Author: Poonam Mani, Department of Obstetrics and Gynecology, Subharti Medical College, Meerut, Uttar Pradesh, India, Phone: +91 9917477661, e-mail:poonam.mani@rediffmail.com

How to cite this article: Mani P, Yadav L, Singh A, et al. Value of Hysteroscopy and Transvaginal Sonography in Endometrial Pathology in Bleeding and Nonbleeding Postmenopausal Women. J South Asian Feder Obst Gynae 2020;12(2):91-95.

Source of support: Nil

Conflict of interest: None
}

\section{Aims and Objectives}

The aim of the present study was to study the value of hysteroscopy and TVS in endometrial pathology in bleeding and nonbleeding postmenopausal women.

\section{Materials and Methods}

A prospective study was conducted in the Department of Obstetrics and Gynecology, Subharti Medical College, Meerut, Uttar Pradesh, India. Postmenopausal patients who came to our outpatient clinic with complaints of PMB, lower abdominal pain, abnormal vaginal

() The Author(s). 2020 Open Access This article is distributed under the terms of the Creative Commons Attribution 4.0 International License (https://creativecommons. org/licenses/by-nc/4.0/), which permits unrestricted use, distribution, and non-commercial reproduction in any medium, provided you give appropriate credit to the original author(s) and the source, provide a link to the Creative Commons license, and indicate if changes were made. The Creative Commons Public Domain Dedication waiver (http://creativecommons.org/publicdomain/zero/1.0/) applies to the data made available in this article, unless otherwise stated. 
discharge, or for a routine gynecological examination between 2011 and 2016 were evaluated. A total of 280 menopausal patients were included in our study with or without PMB. The study was approved by the institutional ethical and research committee board.

Exclusion criteria included patients with vaginal infection; premalignant and malignant conditions of vulva, vagina, cervix, and endocervix; adnexal mass; bleeding diathesis; and patients on hormone replacement therapy (HRT) and tamoxifen. These patients underwent both transvaginal ultrasound examination and hysteroscopy. A biopsy was obtained from all patients during the hysteroscopy procedure.

\section{Transvaginal Ultrasound Examination}

Transvaginal ultrasound examination was done using PowerVision 6000 SSA ultrasound equipment with a $5.0-7.5 \mathrm{MHz}$ transvaginal transducer. Ultrasonographic examination findings were considered normal if a hyperechoic line was observed in the middle of the uterus along with a homogeneous endometrial lining and distinct margin with the myometrium. In postmenopausal patients, a normal endometrium was defined as having a double-wall thickness of $<4$ $\mathrm{mm}$ consisting of a thin basal layer. Abnormalities were defined as follows: endometrial polyp, uterine myoma, atrophy, and placental residual material. In addition, a nonspecific increase in endometrial echogenicity or presence of fluid in the endometrial cavity is classified as nonspecific abnormal finding. Endometrial cancer was suspected in presence of the heterogeneous endometrium with irregular interface between endometrium with or without fluid collection. In invasive uterine cancer, subendometrial halo is lost. The findings were noted and patients were scheduled for hysteroscopy.

\section{Hysteroscopy}

Hysteroscopy was done using a $5 \mathrm{~mm}$ hysteroscope with $30^{\circ}$ optic telescopes (KARL STORZ GERMANY). Hysteromed was used to distend the uterine cavity. Diagnostic sampling, resection, or curettage was done and samples were sent for histological examination. Hysteroscopy appearances were categorized as follows: normal, endometrial polyp, endometritis, atrophy, uterine myoma, malignancy, hyperplasia, disordered proliferative. In addition, nonspecific findings of proliferation and hypertrophy and synechia were categorized as nonspecific findings.

SPSS version 21 was used for statistical analysis. Diagnostic parameters including sensitivity, specificity, positive predictive value, negative predictive value, and diagnostic accuracy of transvaginal ultrasonography (TVU), hysteroscopy, and a combined approach were calculated for the diagnosis of endometrial conditions.

\section{Discussion}

Among 280 cases studied, there were 204 group I patients with postmenopausal bleeding and the rest 76 in group II had come for lower abdominal pain or dragging sensation, backache, and vaginal discharge were included. The mean age of patients was $49.5 \pm 8.63$, and ranged between 42 years and 74 years.

Majority of the patients included had come for PMB $(n=204$, $72.8 \%)$. In 76 patients (27.14\%), a suspicious finding was found on routine gynecological examination or had abnormal TVS findings. In these group II 76 patients, more than $50 \%$ had metabolic syndrome (obesity, diabetes, and/or hypertension). Of these, 13/280 (4.64\%) were nullipara and in all of these the endometrium was thickened (>5 mm). The rest $94.6 \%$ were parous women. For the diagnosis of polyps of any size, hysteroscopy had better sensitivity $(p<0.001)$; however, specificities did not differ $(p=1.0)$.

Of the 204 PMB patients, 117 had abnormal findings. The most common finding was endometrial polyp in 67 (32.64\%). The majority of other studies also report highest incidence of endometrial polyp on hysteroscopy (32.5\% by Dibi et al., $37.6 \%$ by Cordeiro et al. ${ }^{5}$ ).

In our study, 133 cases of endometrial polyp were diagnosed on hysteroscopy of which 115 were $>1 \mathrm{~cm}$ polyp. There were eight submucous myomas diagnosed on hysteroscopy while histopathology showed 12 fibroids. This can be explained by the difficulty in distinguishing small submucous myoma type II from sessile endometrial polyp. There were 7 cases of endometrial hyperplasia and 27 patients had atrophic endometrium. No endometrial pathology was missed on hysteroscopy. Overall, hysteroscopy seems to perform better for most uterine conditions evaluated, although frequencies of endometritis, myoma, cancer, and hyperplasia were relatively low to allow direct comparisons of the two methods. Particularly, its advantage is more prominent when all sizes of the polyps including the smaller ones $(<1 \mathrm{~cm})$ were considered as shown in Table 1. The incidence of myomas in hysteroscopy was $8 / 280(2.85 \%)$ in the present study; out of 280 patients, 12 (4.2\%) were myomas confirmed with histopathology in postmenopausal women with or without bleeding. The accuracy level of hysteroscopy for myoma was less in our study as some submucous myomas were confused with $>1 \mathrm{~cm}$ endometrial polyp in asymptomatic women.

Out of 280 patients, 27 (9.47\%) had atrophy and 100\% confirmed with histopathology; so, in our study $100 \%$ sensitivity and specificity was present. Tables 2 and 3 show diagnostic parameters of the two modalities and the combined approach for the detection of most common pathologies (i.e., endometrial polyp and atrophy). For diagnosing any pathology, hysteroscopy had better specificity $(p<0.001)$, although the two methods did not differ with regard to sensitivity ( $p=0.188$ ). For the diagnosis of polyps of any size, hysteroscopy had better sensitivity $(p<0.001)$; however, specificities did not differ ( $p=1.0)$. On the other hand, hysteroscopy did not have a sensitivity advantage over TVU in diagnosing polyps greater than $1 \mathrm{~cm}(p=0.077)$, although this time hysteroscopy had better specificity $(p<0.001)$. As expected, hysteroscopy had sensitivity advantage in diagnosing atrophy $(p<0.001)$; however, specificities did not differ ( $p=1.0)$. Other pathological conditions had low frequency not allowing direct comparisons between the two methods.

Engin et al. ${ }^{6}$ asses in their study for the assessment of PMB hysteroscopy has high sensitivity and specificity for endometrial space occupying lesions (polyp 100\%, 93\%; fibroid 100\%, 91.8\%). However, for endometrial hyperplasia, hysteroscopy has low sensitivity (75\%); these statistical results correlate with our study as in Tables 1 and 4.

Ultrasonography is of special importance in postmenopausal women. Simple measurement of endometrial thickness by means of TVS in women with PMB could be accurately used to discriminate between normal and pathologic conditions. Several sonographic studies to evaluate a cut-off value for endometrial thickness to exclude endometrial cancer have been published. ${ }^{7}$ An endometrial thickness if $\geq 5 \mathrm{~mm}$ is most commonly seen in the postmenopausal group with malignant changes. ${ }^{8}$

But in our present study until they have significant risk metabolic syndrome (obesity, diabetes, and/or hypertension) for endometrial disease including endometrial hyperplasia and endometrial cancer; as in our study 76 group II had no bleeding 
Table 1: Hysteroscopic findings in all women

\begin{tabular}{llrrr}
\hline Findings & PMB present $(N=204)$ group I & $\%$ & PMB not present $(N=76)$ group II & $\%$ \\
\hline Endometrial polyp & 67 & 32.84 & 66 & 86.84 \\
Submucosal myoma & 06 & 2.94 & 2 & 2.63 \\
Atrophic endometrium & 21 & 10.29 & 6 & 7.8 \\
Proliferative endometrium & 03 & 1.47 & 1 & 1.31 \\
Hyperplasia/cancer & 13 & 6.37 & 1 & 1.31 \\
Endometritis & 7 & 3.43 & - & \\
No abnormal finding & 87 & 42.64 & & \\
\hline
\end{tabular}

PMB, postmenopausal bleeding

Table 2: Diagnostic value parameters of the two methods in diagnosing uterine pathology in PMB group I

\begin{tabular}{lcllll}
\hline All pathology $(n=204)$ & Sensitivity & Specificity & PPV & NPV & $L R(+)$ \\
\hline TVU & $96.0(91.9-98.1)$ & $13.8(7.6-23.2)$ & $71.7(65.8-77.0)$ & $60.0(36.4-80.0)$ & $1.1(1.0-1.2)$ \\
Hysteroscopy & $92.9(88.2-95.9)$ & $41.4(31.1-52.4)$ & $78.3(72.4-83.3)$ & $72.0(57.3-83.3)$ & $1.6(1.3-1.9)$ \\
Combined & $100.0(97.6-100.0)$ & $41.4(31.1-52.4)$ & $79.5(73.9-84.2)$ & $100.0(88.0-100.0)$ & $1.7(1.4-2.0)$ \\
Atrophy $(n=27)$ & & & & & \\
$\quad$ & & & & \\
$\quad$ TVU & $18.5(7.0-38.7)$ & $99.6(97.5-100.0)$ & $83.3(36.5-99.1)$ & $92.1(88.1-94.9)$ & $47.8(5.8-394.1)$ \\
$\quad$ Hysteroscopy & $66.6(46.0-82.8)$ & $99.6(97.5-100.0)$ & $94.7(71.9-99.7)$ & $96.6(93.5-98.3)$ & $172.0(23.9-1238.6)$ \\
$\quad$ Combined & $70.4(49.7-85.5)$ & $99.2(96.9-99.9)$ & $90.5(68.2-98.3)$ & $97.0(93.9-98.6)$ & $90.8(22.3-368.9)$ \\
\hline
\end{tabular}

Numbers in parenthesis denote $95 \%$ confidence intervals. LR(+), positive likelihood ratio; NPV, negative predictive value; PMB, postmenopausal bleeding; PPV, positive predictive value; TVU, transvaginal ultrasonography

Table 3: Diagnostic value parameters of the two methods in diagnosing endometrial polyp in PMB group and non-PMB groups or high-risk groups

\begin{tabular}{llllll}
\hline Polyp any size $(n=133)$ & Sensitivity & Specificity & $P P V$ & $N P V$ & $L R(+)$ \\
\hline TVU & $54.9(46.0-63.4)$ & $84.9(77.9-90.0)$ & $76.0(66.0-83.9)$ & $68.3(61.0-74.7)$ & $3.6(2.4-5.4)$ \\
Hysteroscopy & $82.0(74.1-87.9)$ & $84.9(77.9-90.0)$ & $82.6(74.8-88.4)$ & $84.3(77.4-89.5)$ & $5.4(3.7-8.0)$ \\
Combined & $86.5(79.2-91.6)$ & $78.9(71.4-85.0)$ & $78.2(70.5-84.4)$ & $87.0(80.0-91.9)$ & $4.1(3.0-5.6)$ \\
Polyp $>1 \mathrm{~cm}(n=115)$ & & & & & \\
$\quad$ & & & & \\
$\quad$ TVU & $55.7(46.1-64.8)$ & $88.7(81.9-93.2)$ & $80.0(69.3-87.8)$ & $71.0(63.6-77.5)$ & $4.9(3.0-8.0)$ \\
$\quad$ Hysteroscopy & $86.1(78.1-91.6)$ & $91.5(85.3-95.3)$ & $89.2(81.5-94.0)$ & $89.0(82.4-93.3)$ & $10.1(5.9-17.5)$ \\
$\quad$ Combined & $88.7(81.1-93.6)$ & $85.1(77.9-90.3)$ & $82.9(74.9-88.9)$ & $90.2(83.5-94.4)$ & $6.0(4.0-8.9)$ \\
\hline
\end{tabular}

Numbers in parenthesis denote $95 \%$ confidence intervals. LR(+), positive likelihood ratio; NPV, negative predictive value; PMB, postmenopausal bleeding; PPV, positive predictive value; TVU, transvaginal ultrasonography

Table 4: Histopathology findings in all women

\begin{tabular}{llc}
\hline Finding & Histopathology $(n=280)$ & Percentage \\
\hline Endometrial polyp & 133 & 46.66 \\
Atrophy & 27 & 9.47 \\
Endometritis & 13 & 4.56 \\
Uterine myoma & 12 & 4.21 \\
Hyperplasia & 7 & 2.45 \\
Cancer & 6 & 2.10 \\
No abnormal finding & 82 & 29.29 \\
\hline
\end{tabular}

postmenopausal women, out of 76 women $38 / 76(50 \%)$ had metabolic syndrome and $13 / 76(17.10 \%)$ had nullipara so $51 / 76$ (77.10\%) had significant risk for a typical endometrial hyperplasia however they were polypoidal appearing on TVS required hysteroscopy. Where we found the 66/76 (86.84\%) for inactive polyp. But none of them had endometrial CA confirmed by histopathology as shown in Tables 1 and 4.

Finally, Gerber et al. ${ }^{9}$ were advised to detection of asymptomatic endometrial cancer in screened postmenopausal women offer no prognostic advantage over those patients with cancer who had
PMB (as similar finding in our study groups II). They correlate the bleeding time of symptomatic patients strongly correlated with the tumor stage $(p<0.0001)$, which was very low the 5 -year disease-free survival and overall survival rates were $77 \%$ and $86 \%$ (no bleeding), $83 \%$ and $98 \%$ (<8 weeks), $74 \%$ and $90 \% .{ }^{9}$

Drasler et al. ${ }^{10}$ found the incidence of asymptomatic nonbleed polyp in 169 women were randomly screened postmenopausal women, such high finding also observed in our study 66 in nonbleeding postmenopausal women as shown in Table 4 and Figures 1 and 2.

Lev-Sagie et al. ${ }^{11}$ retrospective study on 82 women with an incidental sonographic finding suspected to be intrauterine polyps was undertaken to assess the histopathologic characteristics of such polyps utilizing operative hysteroscopy. ${ }^{11}$ Such finding 66 women had incidental polyps in our study. It appears that the risk of endometrial carcinoma in postmenopausal women with asymptomatic endometrial polyps is low.

Fernandez-Parra et al. ${ }^{12}$ removed 117 polyps in postmenopausal women without bleeding. None were malignant; they pointed out the importance of distinguishing endometrial carcinoma with polypoid growth from carcinoma arising in a polyp in which case the base and surrounding endometrial tissue must be benign. 


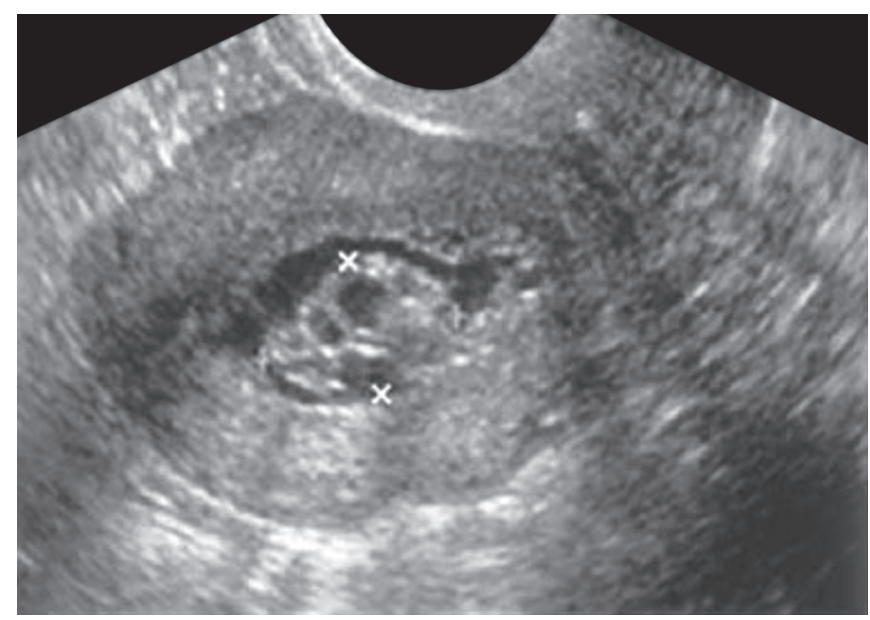

Fig. 1: Transvaginal view of endometrial polyp

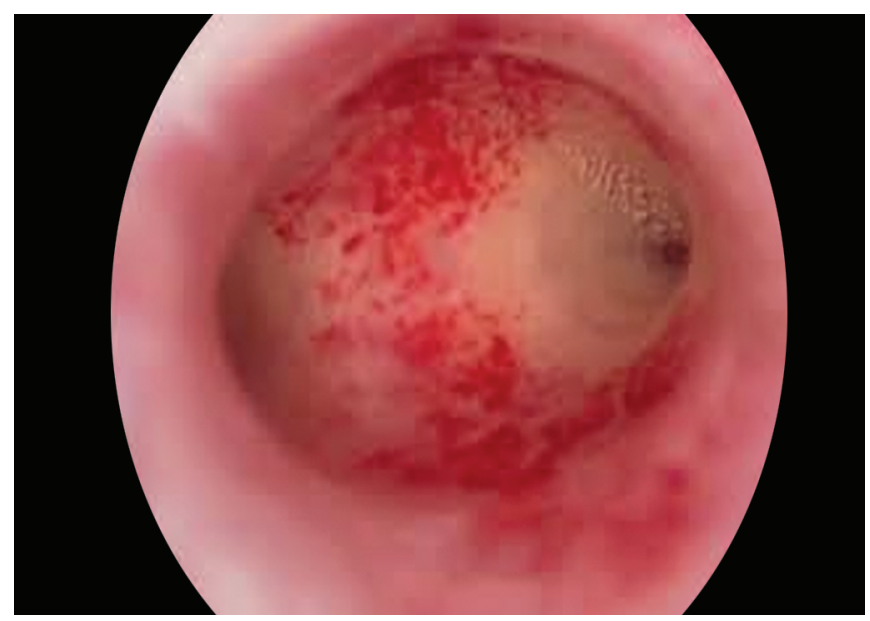

Fig. 3: Hysteroscopy showing thin endometrium with no functional layer but vessels become fragile

Goldstein et al. ${ }^{13}$ - They suggested in their study personally, demoralizing to have promulgated the use of a thin distinct echo in postmenopausal women with bleeding to exclude cancer only to seen clinicians turn that well-studied phenomenon around and assume, therefore, that a thick echo without bleeding should be a cause for cancer and require intervention ${ }^{12}$ (Fig. 3). Therefore in our study, the evaluation of asymptomatic findings, such as abnormal endometrial thickness, may permit the detection of pre-malignant conditions and earlier detection of endometrial carcinoma. Intrauterine polyps undergo the same evaluation although their malignant potential remains unclear.

\section{Conclusion}

However, as clinician I believe it has been and continues to be appropriate to evaluate patients on a case-by-case basis, if someone has asymptomatic endometrial thickening on TVU and also has significant risk for endometrial disease (obesity, history of PCOS, diabetes, etc.); in these patients, endometrial evaluation may in fact still be appropriate.

It has some technical limitations as well as timing sensitivities that must be appreciated for effective use. Finally, an incidental

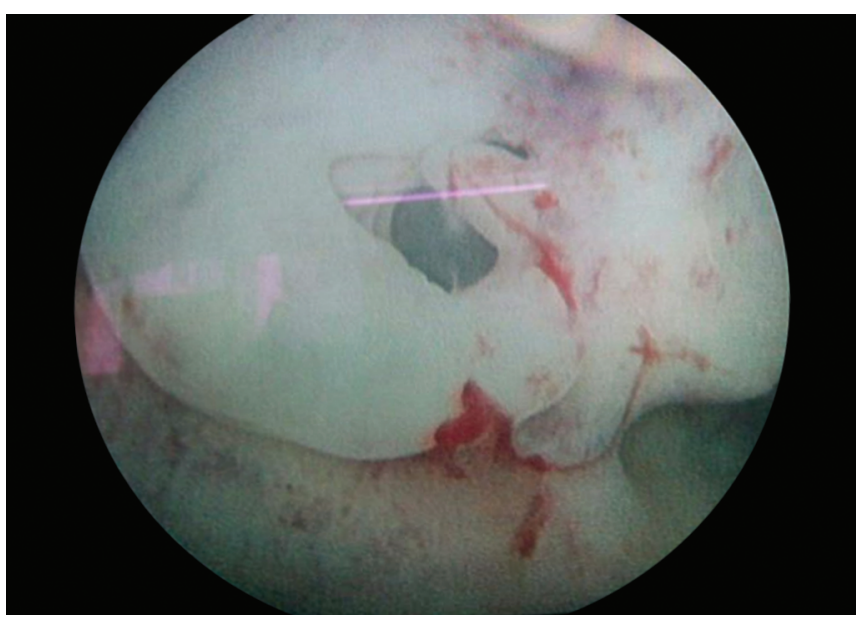

Fig. 2: Hysteroscopic view of asymptomatic endometrial polyp

finding of a thick endometrium on TVS in postmenopausal women is common ( 54 out of $76 ; 71.05 \%$ ), and absence bleeding does not require automatic intervention.

\section{Clinical Significance}

My concern has been that many clinicians thoughts that because an endometrial echo $4 \mathrm{~mm}$ or less in patients with bleeding than this in any patient required evaluation, this is simply not true, never validated, and much more common than previously thought as in our study polypoid tissue mistaken for a polyp might be malignant but none of polyp in asymptomatic postmenopausal women had malignant with histopathology, such an approach would spare the other any intervention and its risks, discomfort, and expense. But we have to evaluate significant high-risk factors for endometrial carcinoma.

\section{Compliance with Ethical Requirements}

An informed consent was obtained from all patients for being included in the study. As this project was registered and the study was approved by the ethical and research review board.

\section{References}

1. Evaluation and management of postmenopausal bleeding. India menopause society Guideline number 4: 2010.

2. Ambreen A, Siddiqi KJ, Atq F, et al. Hysteroscopy: 3 years experience at a teaching hospital. Arm king Edward Med Coll 2007;13:122-123.

3. Wolfman W, Leyland N, Heywood M. Asymptomatic endometrial thickening. clinical practice guideline 249. J Obstet Gynaecol Can 2010;32(10):990-999. DOI: 10.1016/S1701-2163(16)34690-4.

4. Dibi RP, Zrttler CG, Pessini SA, et al. Tamoxifen use and endometrial lesions:hysteroscopichistological and immunnohistochemical findings in postmenopausal women with breast cancer. Menopause 2009;16(2):293-300. DOI: 10.1097/gme.0b013e31818af10a.

5. Cordeiro A, Condecor R, Sousa F, et al. Office hysteroscopy after ultrasonographic diagnosis of thickened endometrium in postmenopausal women. Gynecol Surg 2009;6(4):317-322. DOI: 10.1007/s10397-009-0485-3.

6. Korkmazer E, Solak N, Ustunyurt E, et al. Role of hysteroscopy in evaluation of postmenopausal bleeding hysteroscopy in menopause. 
Turkiye Klinikleri Jinekoloji Obstetrik 2015;25(3):141-145. DOI: 10.5336/ gynobstet.2014-42527.

7. Coleman B, Arger P, Grumback K, et al. Transvaginal and transabdominal sonography: prospective comparison. Radiology 1988;168(3):639-645. DOI: 10.1148/radiology.168.3.3043545.

8. Osmers R, Volksen M, Schaver A. Vaginal sonographic detection of endometrial cancer in postmenopausal women. Int J Gynecol Obstet 1990;32(1):35-37. DOI: 10.1016/0020-7292(90) 90979-U.

9. Gerber B, Krause M, Muller $\mathrm{H}$, et al. Ultrasonographic detection of asymptomatic endometrial cancer in postmenopausal patients offers on prognostic advantage over symptomatic disease discovered by uterine bleeding. Eur J Cancer 2001;37(1):64-71. DOI: 10.1016/S09598049(00)00356-7.
10. Dreisler $\mathrm{E}$, Sorensen $\mathrm{SS}$, Ibsen $\mathrm{PH}$, et al. Value of endometrial thickness measurement for diagnosing focal intrauterine pathology in women without abnormal uterine bleeding. Ultrasonal Obstet Gynecol 2009;33(3):344-348. DOI: 10.1002/uog.6256.

11. Lev-Sagie A, Hamani $Y$, Imbar $T$, et al. The significance of intrauterine lesion detected by ultrasound in asymptomatic postmenopausal patients. BJOG 2005;112(3):379-381. DOI: 10.1111/j.1471-0528.2004.00444.x.

12. Fernandez-Parra J, Rodriquez Oliver A, Lopez Cariado S, et al. Hysteroscopic evaluation of endometrial polyps. Int J Gynaecol Obstet 2006;95(2):144-148. DOI: 10.1016/j.ijgo.2006.07.007.

13. Goldstein SR. The endometrial echo revisited have we created a monster? AM J Obstet Gynecol 2004;191(4):1092-1096. DOI: 10.1016/ j.ajog.2004.05.017. 